\title{
Fabula burlesca de Jupiter y Europa de Manuel de Pina
}

Michèle Guiraud Université de Nancy 2 - França

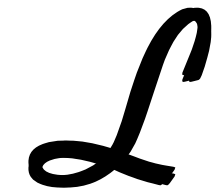

a biographie de Manuel de Pina, dont le nom figure parfois dans les anthologies de texte ou les histoires littéraires, reste encore à être approfondie. Rares sont en effet les informations que nous avons pu trouver concernant cet auteur. Tout comme António Serrão de Castro, ${ }^{1}$ Manuel de Leão ${ }^{2}$ ou encore D. Joseph de la Veja, ${ }^{3}$ il fait partie de ces érudits séfardis ${ }^{4} \mathrm{du} X \mathrm{XII}{ }^{\mathrm{e}}$ siècle dont l'impact littéraire et parfois même le rôle politique ont été méconnus des historiens. Ces auteurs partagèrent le même goût pour la poésie baroque et, en raison de leur situation de nouveaux-chrétiens, connurent les rigueurs de l'Inquisition. Manuel de Pina, né à Lisbonne en $1616,{ }^{5}$ eut la chance de quitter le Portugal comme bon nombre de ses coreligionnaires. Si l'on n'a aucune date concernant cet exil, on sait que, dans un premier temps, il s'installa à Bruxelles ${ }^{6}$

\footnotetext{
${ }^{1}$ Voir, GUIRAUD, 1997, p. 5-39; GUIRAUD, 2002, p.189-203; GUIRAUD, 2010, p. 35-57.

${ }^{2}$ Voir, GUIRAUD, 2007, p. 57-65.

${ }^{3}$ Voir, GUIRAUD, 2008, p. 289-302.

${ }^{4}$ Voir, DÍAZ ESTEBAN, 1994. Plus particulièrement l'article de Javier Huerta Calvo consacré à Manuel de Pina, p. 215-228.

5 THE JEWISH ENCYCLOPEDIA, p. 44.

${ }^{6}$ SCHOLBERG, 1967, p. 60.
} 
où il fréquenta Juan de Prado, D. Nuno de Figueroa et Juan de Faria, autres Juifs comme lui ayant fui la péninsule Ibérique. Ces derniers, également férus de poésie, composèrent plusieurs poèmes en son honneur. Manuel de Pina vécut ensuite à Amsterdam $^{7}$ où il prit le nom de Jacob de Pina. ${ }^{8}$ Si l'on ne connaît pas la date de sa mort, on sait pour le moins qu'il était encore en vie en 1660, année où il écrivit un poème intitulé: "Cancho fúnebre na lamentable falta do... Haham Saúl Levi Mortera". 9

\section{Présentation de l'œuvre}

L'ouvrage sur lequel nous avons travaillé fait partie de la Bibliothèque Alfonso Cassuto et se trouve à la UB d'Amsterdam, numéro 0119960151. Un autre exemplaire est signalé à la Bibliothèque nationale de Madrid, sous le numéro R.11.887. ${ }^{10}$ Les deux ouvrages portent le même titre, à savoir, Chanças / del ingenio, y dislates / de la Musa. / Dirigidas al muy noble, y Magnífico / Sñor, / Jerónimo Nuñez / de Acosta, / Cavallero hijodalgo de la caza de /su Magestad. el Rey / Don Juan IV. de Portu-/gal, y su agente en / los estados de / Holanda. En bas de page est écrit: Compuestas por Manuel De / Pina, Natural de la / Insigne ciudad de / Lisboa. / Año 1656. L'œuvre se compose d'une première dédicace de l'auteur adressée à Jerónimo Nunes da Costa - vraisemblablement son mécène, p. III-VII, suivie d'une autre dédicace adressée au lecteur, p. IX-XIII, de plusieurs poèmes écrits par ses amis de Bruxelles, p. XIV-XXVI, d'une série de poèmes, soit trente-neuf,

\footnotetext{
${ }^{7}$ F: \Israël Salvator Révah definição de Israël Salvator Révah e sinónimos de Israël Salvator Révah (espanhol).mht

${ }^{8}$ SCHOLBERG, 1967, p. 61.

${ }^{9}$ SCHOLBERG, 1967, p. 62.

${ }^{10}$ SCHOLBERG, 1967, p. 63.
} 
p. 1-94, et d'une comédie burlesque, p. 95-134. Si cette dernière, intitulée La mayor hazaña de Carlos VI, a été analysée, ${ }^{11}$ en revanche, l'ensemble des poèmes n'a fait l'objet, à notre connaissance, d'aucune étude approfondie.

L'auteur dédie son œuvre à Jerónimo Nunes da Costa, ${ }^{12}$ gentilhomme de la maison du Roi, qui était alors l'agent de D. Jean IV dans les Provinces-Unies. Celui-ci occupera encore les mêmes fonctions durant le règne de D. Pierre II. La famille Nunes da Costa, en effet, rendit de nombreux services au Portugal. ${ }^{13}$ Jerónimo Nunes da Costa, de son vrai nom, Moisés Curiel, était le fils de Duarte da Costa, auquel l'auteur fait référence. Celui-ci était également l'agent de D. Jean IV mais en Allemagne.

\section{Fabula burlesca de Jupiter y Europa}

Cette composition poétique figure aux pages 1-8. Elle est rédigée en espagnol. L'auteur s'exprimait dans les deux langues, portugais / espagnol, mais l'espagnol offrait souvent une plus large diffusion. Nous avons choisi cette composition car elle nous semble être un exemple probant du ton et du style de l'auteur. Cherchant à faciliter la lecture des extraits donnés, nous avons actualisé le texte en séparant les mots mais nous avons tenu à préserver les majuscules ainsi que l'orthographe ancienne afin de ne pas l'altérer. Ce poème, comme tous les autres, porte les marques d'une tradition littéraire et correspond parfaitement au goût de la société portugaise du XVII ${ }^{\mathrm{e}}$ siècle.

${ }^{11}$ Voir, SCHOLBERG, 1967, p. 64-80.

12 GUIRAUD, 2007, p. 58-59.

${ }^{13}$ Voir, GUIRAUD, 1992. 
Comme beaucoup d'écrivains séfardis, ne voulant pas rompre avec toute culture hispanique, Manuel de Pina transporta les diverses formes de vers et de strophes espagnols dans la poésie néo-hébraïque. Dans une continuité avec leur passé, ces intellectuels sentirent aussi le besoin de lire et d'échanger les idées dans des Cercles comme ils le faisaient dans leur terre natale.

En Europe, la société du XVII ${ }^{\mathrm{e}}$ siècle vivait une crise à tous les niveaux, à la fois politique, économique, sociale, religieuse et scientifique. Les nouveaux-chrétiens de la péninsule Ibérique, lesquels avaient dû quitter leur pays, savaient plus que toute autre personne ce que pouvait signifier l'infortune et le changement. Dans ce contexte général, l'art de la Renaissance, qui prônait l'harmonie et l'optimisme ne correspondait plus à la dure réalité du moment. Le mouvement baroque offrait une réalité complexe, faite de contrastes, montrant la fuite du temps et l'obsession de la mort.

Si les cours baroques choisissent, néanmoins, une certaine évasion à travers les fêtes somptueuses, nombreux sont aussi les hommes de lettres, tel Manuel de Pina, qui optent pour la dérision. Ils composent des poèmes en parodiant les thèmes et le style hérités de la Renaissance. ${ }^{14}$ Ainsi, utilisent-ils toujours la fable mais d'une manière différente. La fable mythologique apparaît dans le monde antique comme la manière poétique de raconter une histoire. La langue classique utilise le terme de fable pour désigner les récits de la mythologie antique, à savoir les aventures des dieux et des héros antiques. ${ }^{15}$ En réaction contre les images

${ }^{14}$ Voir par exemple, en Catalogne, [F: $\backslash$ Barroco_ Culturcat_ Generalitat de Catalunya.mht]

${ }^{15}$ GALLEGO GIMÉNEZ, p. 6. 
élaborées à la Renaissance, la fable devient burlesque. Luis de Góngora traita de manière burlesque les thèmes mythologiques, par exemple, en 1618, dans la Fábula del Píramo y Tisbe ${ }^{16}$ et le modèle fut fixé par Polo de Medina avec la Fábula de Apolo y Dafne, en 1634.

Le mythe de l'enlèvement d'Europe par Jupiter fut une source d'inspiration pour la littérature grecque et latine. Il inspira par la suite de nombreuses œuvres tant sur le plan littéraire qu'artistique. Il semble être à la mode au début du XVII ${ }^{e}$ siècle puisqu'on le trouve utilisé par plusieurs auteurs de la péninsule Ibérique comme, par exemple, en Catalogne, avec Francesc Vicent Garcia qui compose la Faula de Júpiter $i$ Europa, de148 vers. ${ }^{17}$

Un bref résumé du mythe: Europe était la fille du roi Agénor, roi de Phénicie et sœur de Cadmos. Alors qu'elle était en train de jouer sur la plage avec ses compagnes, Zeus la voyant s'éprit d'elle. Pour la séduire, il se métamorphosa en taureau blanc et se prêta aux jeux et caresses des jeunes filles. Europe s'enhardit et monta sur son dos. C'est alors que Zeus l'emporta et traversa la mer jusqu'en Crète. De cette union naquirent Minos, Rhadamanthe et Sarpédon. Cet épisode marque le début de la dynastie crétoise. Les romains ont assimilé Zeus à Jupiter.

Manuel de Pina, qui avait connaissance vraisemblablement des fables burlesques déjà écrites à son époque que ce soit sur ce mythe ou sur les amours de Jupiter et Danaé ${ }^{18}$ ou encore sur

${ }^{16}$ Voir, JURADO DOMÍNGUEZ, febr. 2007.

${ }^{17}$ BARRERA LÓPEZ, 1979, p. 3.

${ }^{18}$ Voir, TRAVER VERA, 1996, p. 211-234. Par exemple, Francesc Vicent Garcia a composé Faula de Júpiter i Dànae. 
celles d'Apollon et de Daphnée, ${ }^{19}$ se place, dès son titre, dans la démystification. Il affiche ainsi sans ambigüité la négation du mythe et adopte une attitude critique par rapport aux topiques littéraires. On est loin, en effet, de la tradition pétrarquisante par rapport à la représentation de l'amour.

La fable raconte une histoire que l'on peut diviser en trois moments: la présentation des personnages et la localisation; le déroulement de la scène avec la rencontre et l'enlèvement de la jeune fille; la conclusion (les conséquences de l'acte). Pour replacer le récit dans la réalité, l'auteur n'hésite pas à insérer le style direct des échanges entre les personnages et à s'adresser directement au lecteur. Il utilise des expressions familières, recours communs à ce type de poésie burlesque, et, aux termes poétiques, il mêle un vocabulaire banal qui, sorte d'antithèse, accentue le contraste. À cela s'ajoute, l'utilisation constante du double sens des mots.

Le ton burlesque est donné dès le début du poème, la jeune fille Europe est "tan bizarra" (si gracieuse) que "Que siendo parte del Mundo, / Todas las del Mundo abarca" (qu'étant partie du monde / elle comprend et/ou embrasse toutes les parties du Monde). En effet, le nom de celle-ci signifierait "à l'aspect large ou large terre". La thématique sexuelle est donnée par le verbe "abarca". Hérodote assimilait 1'Europe à la Grèce, en opposition à 1'"Afrique" et 1'"Asie". Ces deux dernières, compagnes de la jeune fille, loin d'être

\footnotetext{
${ }_{19}$ Par exemple, dans la péninsule Ibérique, Faula de Apolo i Dafne de Francesc Vicent Garcia ou Fábula de Apolo y Dafne de Polo de Medina. On peut citer également, en France, une comédie mythologique, Iphis et Lante, tirée des Métamorphoses d'Ovide, écrite en 1634, par Isaac de Bensserade ainsi qu'une comédie en musique, en 1650, Les Amours d'Apollon et de Daphnée, composée par Charles Coypeau d'Assoucy.
} 
d'ascendance divine sont "dos vezinas" (deux voisines). L'aparté entre parenthèses "(Ay quien dize a comer natas)" (certains disent pour manger des gâteaux à la crème / offrir le meilleur de soi aux autres) accentue la touche populaire de ce premier tableau.

En lien au thème énoncé, se développe, la thématique florale. Thème fréquemment employé dans la poésie baroque avec l'idée de profusion, d'exubérance, de printemps et de jeunesse. Mais ici, la floraison glisse très vite vers la défloraison. On trouve ainsi le champ lexical de "flores" (fleurs), "guirnaldas" (guirlandes), "mil flores" (mille fleurs) jusqu'à la perte de la virginité "de su flor Jupiter usa" (Jupiter use de sa fleur). L'auteur, par dérision, semble vouloir rester fidèle au récit d'Ovide: "elle s'approche et lui présente des fleurs [...]. Elle pare ses cornes de guirlandes de fleurs". ${ }^{20}$ L'expression qui introduit la deuxième scène: "A darse un verde" place le lecteur dans une atmosphère irrévérencieuse avec l'idée de "faire quelque chose à satiété" que renforce: "Entre lo roxo y lo verde" (avoir une aventure).

L'harmonie et la paix pourraient se dégager de cet espace bucolique du "campo" (campagne), la présence du bétail n'est pas sans rappeler le cadre des églogues. Néanmoins, dans un procédé de monde à l'envers, ce paysage idyllique, qui dans la tradition était en consonance avec les sentiments amoureux, ne va susciter que désir brutal. Dans la présentation des personnages, le dieu est tout de suite associé au mot "luxuria" (exubérance, abondance mais aussi excès, luxure) et à celui de "chispa" (étincelle). L'auteur joue sur le double sens du mot, rappelant que Jupiter a pour attribut la foudre et qu'il plaît tout de suite à la jeune fille. Celle-ci le remarque parmi tous les autres en raison de sa couleur "un blanco toro" (un taureau

${ }^{20}$ OVIDE. Métamorphoses, Livre II. 
blanc). Dans le culte à Jupiter, les Romains offraient une chèvre, une brebis et un taureau blanc dont on avait eu soin de dorer les cornes. Les adjectifs employés par l'auteur traduisent la psychologie des deux personnages. Europe apparaît "apacible" (paisible), "asustada" (effrayée) tandis que Jupiter, héros rabaissé au niveau du grotesque, n'est plus que le "reboçado en la maula" (pané, dans la tromperie), le "socarrón" (sournois). Avec les verbes "gozar" (jouir), "apetecer" (avoir envie), on entre désormais dans la trivialité et la couleur blanche, symbole de pureté, dérive vers l'expression "siendo / Ella el blanco, de sus ancias" (étant / Elle la cible de ses envies). La relation à l'aimée dans la tradition classique, où la nymphe est décrite en partant du haut vers le bas, se présente tout le contraire: "besar sus plantas" (baiser ses pieds), "lame sus manos de plata" (lèche ses mains d'argent), "ablandarla el pecho" (adoucir sa poitrine), "el ardor de sus ojos" (l'ardeur de ses yeux). Les allitérations en "l" avec l'amplification dans le choix des mots: "lame" (lèche), "el lamedor" (le léchage), l'association des deux substantifs "lameduras besos" (actions et effets de lécher, baisers) entretiennent l'équivoque. Ces termes utilisés, contraires à l'amour chevaleresque, apparaissent comme un contrepoint aux amours d'Angélique et Médor dont il est fait référence: "Medoro" - personnage de l'Orlando furioso de l'Arioste -, "el agradecido amante" (l'amant reconnaissant) d'Angélique qui soigna ses blessures. Enfin, le parallélisme dans les vers: "Quiere hazer juego de toros / S y el, de sortija, y de lança" (elle veut faire une course de taureaux / et lui, de bague et de lance) et les allusions qui s'ensuivent provoquent le rire. Zeus était vénéré, en effet, dans toute la Grèce et, en son honneur, on célébrait de grandes fêtes accompagnées de compétitions et de jeux. L'expression "courir une lance" se dit au jeu de bague de la course à la lance pour décrocher et enlever une bague. L'association des mots "lengua de â vara" et "lança" nous autorise à penser au proverbe populaire: "Coup de langue est souvent pis que coup de lance". 
L'auteur semble prendre plaisir à manipuler les équivoques à travers les expressions familières voire triviales de la jeune fille: "guevo" (sexe). Il joue constamment sur la polysémie: "quieres sal" (sel / charme) / Yo te passare por agua (je te passerai à l'eau / je te baptiserai). On assiste, de plus, à un crescendo donné à la fois par le vocabulaire et les sonorités: "Bozes davan las dos Ninfas" (les deux nymphes criaient en pleurant, "Grita Europa, y no le valen / los sollozos, y las ancias" (Europe crie et ne lui servent à rien / les pleurs ni les envies), "La hermosa ninfa llorava" (la belle nymphe pleurait), "Teme Europa" (Europe a peur), "Dado aperros yva el toro / Como si fuera en la plaça" (le taureau criait / Comme s'il était dans l'arène). L'action est rendue par le jeu des oppositions avec les idées de "jeter, lancer" mais aussi d"'attacher": "Con la presa al mar se arroja" (avec la prise à la mer il se jette), "Se quiere, echar con la carga" (il veut se débarrasser de sa charge), "echar perlas à puercos" (donner des perles aux pourceaux), "Ella agarrada de un cuerno, / El asido de sus faldas" (elle accrochée à une corne, / Lui accroché à ses jupes). Manuel de Pina parodie le style héroïque de l'épopée maritime : "Gallarda sulca las aguas, / Y con llevarle à la vela / Va con dos remos por banda" (Gaillarde elle sillonne les eaux, / Et l'amenant à la voile / Va avec deux rames de côté du bateau comme ceinture); la métaphore de la voile est la robe d'Europe qui flotte au vent. De même, l'image de la "Bestia, pero bestia mala" (bête, mais sale bête) est-t-elle développée pour finir avec l'image du pourceau à laquelle s'oppose la métaphore de Jupiter désigné comme "el novio de Xarama" (le fiancé de Xarama) - les taureaux les plus braves étant, selon la tradition, ceux qui vivaient sur les bords du Tage et du Xarama. Jupiter est converti en personnage vulgaire, réclamant une "fabor" (relation sexuelle occasionnelle), demande que l'auteur tourne en ridicule en jouant sur les phonèmes: "No del Cielo de sus glorias / Mas del suelo de sus nalgas" (non du ciel de ses gloires / Mais du bas de ses fesses). L'idée de relation sexuelle se 
poursuit avec les jeux de mots sur "apelo/ Pelo": "Pero ya no ay mas apelo / Que a los pelos de la manta, / Dexa que te ocupe toro / Si no quieres quedar vaca" (Mais maintenant il n'y a pas d'autre appel / Qu'aux poils de la couverture / Laisse que le taureau s'occupe de toi / Si tu ne veux pas rester une vache) et la "esphera de la cama" (la sphère du lit) remplace la sphère céleste. Europe est comparée à Io, autre maîtresse de Jupiter que celui-ci transforma en vache.

La troisième scène se déroule en Crète. L'idée de "quitter, enlever", se poursuit avec l'assouvissement du désir pour le taureau et la désillusion d'Europe: "Temia del laberyntho / La salida, no lo entrada / La cabellera de cuerno / Se quita el Dios de las trampas / Y se desuella, el pellejo" (Elle craignait du labyrinthe / La sortie, non l'entrée / La chevelure de corne / le Dieu des tromperies l'enlève / Et s'enlève la peau). Le verbe "desollar" signifie à la fois "enlever la peau à un animal" et au sens figuré "ruiner". L'auteur se plaît à détourner le sens de certains mots comme "entrée / sortie" du labyrinthe et il multiplie ainsi les allusions sexuelles: "diran lenguas malas / Que le à quitado el pellejo" (les mauvaises langues diront / Qu'il lui a enlevé sa peau, au sens de virginité), "Y es, en cierto modo, estafa" (Et c'est, d'une certaine façon, une demande de quelque chose de valeur par artifices ou tromperies), "la luxuria asomada" (la luxure qui se laisse voir). Le dénouement est le retour à la triste réalité: "Torear, es galanteo / Pero el ser toro es infamia" (tauréer est galanterie / Mais être taureau est infamie) et l'intervention de l'auteur constitue une mise en garde et une première morale à la fable: "No he visto transformación / Mas bestia, y mas escusada, / Que dexa el que se haze toro / Para el tiempo en que se casa" (Je n'ai pas vu de transformation / Plus bête et plus différente de l'habituelle, / Que montre celui qui se fait taureau / Au moment où il se marie). Ce retour à la réalité marque une pause, et un changement de ton, pour dénoncer la fausseté des attitudes humaines. Mais l'action reprend et 
l'auteur adopte à nouveau son ton burlesque, s'appuyant sur le champ lexical du jeu et du bétail: "Y con sus onze de oveja" (Et pour donner à entendre que quelqu'un se mêle de ce qui ne le regarde pas), "amenaça" (crie pour mener le troupeau), "Fullero [...] / Y à la primera que juega / Haziendo flux se la clava" (Trompeur / Et à la première qu'il joue / Ayant les cartes de son côté il la lui plante". Au moment crucial de la relation amoureuse, on retrouve la thématique florale mais ridiculisée: "Malvas, y paja le sirven / De lecho a la desdichada, / Y aun que en malvas no ha nacido / Oy se ha dormido en las pajas" (Mauves et herbe lui servent / De lit à la malheureuse / Et bien qu'elle ne soit pas d'humble naissance / Aujourd'hui ellen'a pas été attentive pour profiter des occasions). Description, on ne peut plus antipoétique, où l'odeur de la transpiration se mêle au parfum habituel des jasmins et où les genêts, plantes peu poétiques avec lesquelles on fait les balais, constituent la base du lit nuptial: "Dava el copioso sudor / Da sus cuerpos tal fragancia / Que el lecho humilde parece / De jasmines, y retamas" (Un tel parfum se dégage de leurs corps / Que l'humble lit semble / De jasmins, et genêts); l'amour chevaleresque a laissé place à la seule relation sexuelle: "Que en afloxando el amor / El apetito adelgaça" (Car en effleurant l'amour / L'appétit s'amenuise), le verbe "afloxar" signifiant "passer au tamis une céréale pour obtenir la fleur", d'où l'idée ici de "déflorer". Jupiter est relégué au rang d'humain grossier: "Al Cielo Jupiter sube / De la cama mas cercana, / Que sus cielos, y sus glorias / Siempre an parado en la cama" (Jupiter monte au Ciel / Du lit le plus proche / Car ses cieux et ses gloires / Ont toujours fini au litt ${ }^{21}$ ); le mouvement ascendant et descendant traduit le comique de la situation.

${ }^{21}$ Voir par exemple, GARCIA MASEGOSA, 1998. 
Une morale, en guise d'épilogue, parachève cette rencontre amoureuse:

"Huye el Dios rufian en fin

Y deja Europa burlada,

Para que en ella escarmienten,

Las solteras, y casadas.

Y sepan que el mejor campo

De la muger es la casa

Por que siempre las salidas

Suelen parar en entradas."

(Le Dieu ruffian s'enfuit enfin

Et laisse Europe trompée,

Pour que par cet exemple se corrigent,

Les célibataires, et les femmes mariées.

Et qu'elles sachent que la meilleure campagne

De la femme est la maison

Parce que les sorties toujours

Finissent habituellement en entrées.)

On retrouve dans cette conclusion quelques-uns des mots clefs et leur contraire: "campo" espace ouvert / "laberyntho", "casa" espace fermé; "entrada" entrée / "salida" sortie. Le décor enchanteur s'est refermé sur celui du désenchantement. Ainsi, Manuel de Pina, à travers la parodie de la thématique amoureuse, met-il en garde la femme, victime de l'hypocrisie du jeu amoureux. La drôlerie fut pour beaucoup de séfardis comme lui le moyen de détourner ou d'affronter la triste réalité. Pourtant, malgré le réalisme et la sincérité de son auteur, l'œuvre Chanças fut loin d'être appréciée puisqu'elle fut interdite de publication, et ce à trois reprises, par la communauté judéo-portugaise d'Amsterdam, ${ }^{22}$ en raison de son caractère irrévérencieux.

${ }^{22}$ REVAH, p. LXXIV-XCI. 
Ce poème ne donne qu'un faible aperçu de la valeur littéraire de l'auteur. Dans un exercice de style éminemment baroque, Manuel de Pina, à l'instar d'un Quevedo, mêlant le culte au populaire, le grave au burlesque, a su traiter non sans une certaine profondeur la psychologie de l'âme humaine.

\section{Bibliographie}

BARRERA LÓPEZ, Trinidad. La fábula burlesca de Júpiter y Io, de Juan del Valle y Caviedes. In: Anales de literatura hispanoamericana, n 8, p. 3, 1979.

[F: \Barroco_C Culturcat_Generalitat de Catalunya.mht]

DÍAZ ESTEBAN, Fernando. Los judaizantes en Europa y la literature castellana del siglo de oro. Madrid: Letrúmero S.L., 1994.

GALLEGO GIMÉNEZ, Cristina. Les faules mitològiques burlesques catalanes dins de la modernitat barroca. Una particular apropiació de la mitologia clàssica, p. 6. [http://openaccess.uoc.edu/webapps/o2/ bitstream/10609/358/1/36192tfc.pdf]

GARCIA MASEGOSA, Antonio. Los amores humanos de Zeus. Vigo: Servicio de Publicaciones de la Universidad de Vigo, 1998.

GUIRAUD, Michèle. Jeu de canne à Lisbonne (1666). Étude d'un poème d'António Serrão de Castro. Quadrant, Université PaulValéry-Montpellier III, n. 14, p. 5-39, 1997.

GUIRAUD, Michèle. Arcs de triomphe érigés en la ville de Lisbonne (1666); extrait d'un poème inédit d'António Serrão de Castro, Espaces de l'image. Revue XVI ${ }^{e}$-XVII ${ }^{e}$, Nancy 2, v. 3, p.189-203, 2002.

GUIRAUD, Michèle. Fêtes baroques à Lisbonne: compositions poétiques d'António Serrão de Castro (1666). In: GUIRAUD, Michèle (Dir.). Fêtes et traditions dans le monde luso-hispanophone, Mélanges en l'honneur de Nicole Fourtané. Nancy: Presses Universitaires de Nancy, 2010. 
GUIRAUD, Michèle. Fêtes de cour à Lisbonne: Triumpho Lusitano, applausos festivos (1668) de Manuel de Leão. In: GUIRAUD, Michèle. La mémoire historique dans les Lettres et les Arts de l'aire romane, P.R.I.S.MI., Université Nancy 2, n. 8, p. 57-65, 2007.

GUIRAUD, Michèle. Mémoires d'une ambassade portugaise à Mannheim: Alientos de la verdad en los clarines de la Fama (1687) de D. Joseph de la Veja. In: FOURTANÉ, Nicole; GUIRAUD, Michèle (Dir.). Mémoire et culture dans le monde luso-hispanophone. Nancy: Presses Universitaires de Nancy, 2008.

GUIRAUD, Michèle. Fêtes de cour à Lisbonne: Triumpho Lusitano, applausos festivos (1668) de Manuel de Leão. In: La mémoire historique dans les Lettres et les Arts de l'aire romane. P.R.I.S.MI., Université Nancy 2, n. 8, p. 58-59, 2007.

GUIRAUD, Michèle. Marchands-banquiers juifs dans le Portugal de la deuxième moitié du XVII ${ }^{e}$ siècle. In: Actes $d u$ Colloque Les juifs et l'économique. Miroirs et mirages, Toulouse, PUM, 1992.

[F: Israël Salvator Révah definição de Israël Salvator Révah e sinónimos de Israël Salvator Révah (espanhol).mht]

JURADO DOMÍNGUEZ, Purificación. Fábula de Píramo y Tisbe, Luis de Góngora. Comentario del texto y comparación con otras versiones. Revista Digital, n. 28, v. 1, feb. 2007.

OVIDE. Métamorphoses, Livre II.

REVAH, I.S. Les écrivains Manuel de Pina et Miguel de Barrios et la censure de la communauté judéo-portugaise d'Amsterdam. In: Tesoro de los judios sefardies, Estudios sobre la historia de los judios sefardies y su cultura, v. VIII, Jerusalem-Israel, p. LXXIV-XCI.

SCHOLBERG Kenneth R. Manuel de Pina y La May, Thesaurus, Tomo XXII, Núm. 1, 1967, p. 64-80.

THE JEWISH ENCYCLOPEDIA. New York-London, 1945. t. X.

TRAVER VERA, Ángel Jacinto. El mito de Dánae: interpretación y tratamiento poético desde los orígenes grecolatinos hasta los Siglos de Oro en España. Cuadernos de filología clásica: Estudios latinos, n. 11, p. 211-234, 1996. 


\section{Resumo}

Manuel de Pina faz parte daqueles eruditos sefardins do século XVII cujo impacto literário e às vezes o papel político foram desconhecidos pelos historiadores. Atravès dum poema Fabula burlesca de Jupiter y Europa, enxerto da obra dela Chanças del ingenio, composta em 1656, analisaremos a maneira burlesca de tratar do mito e da temática do amor.

\section{Résumé}

Manul de Pina fait partie de ces érudits séfardis du XVII siècle dont l'impact littéraire et parfois le rôle politique ont été méconnus des historiens. À travers un poème Fabula burlesca de Jupiter y Europa, extrait de son œuvre Chanças del ingenio, composée en 1656, nous analyserons la façon burlesque de traiter le mythe et la thématique amoureuse. 